\title{
Multi-Beacons Energy-efficient Geographical Routing for Low Rate Wireless Personal Area Networks
}

\author{
Mu Yan \\ Department of Electronic, Information and \\ Communication Engineering, \\ Konkuk University
}

\author{
Younggoo Kwon \\ Department of Electronic, Information and \\ Communication Engineering, \\ Konkuk University
}

\begin{abstract}
IEEE $802.15 .4 \mathrm{a}$ standard based geographical routing provides low data rate connectivity at a very small power consumption and low complexity to inexpensive devices. The existing geographical relies on finding the least power consuming path by creating and maintaining routing tables. Beacon packets are used for computing power and energy consumption between two nodes. This power and energy consumption information is used for creating and updating the routing tables at various nodes. For every change in the data transmission conditions, multiple beacon packets are exchanged between various nodes for updating the routing tables. The network time and power consumption during this interval is undesired and hence must be minimized. In this paper, multiple beacons based energy efficient geographical (MbIEG) routing scheme for IEEE 802.15.4a standard is presented. The proposed scheme uses three types of beacon packets of different bit length. The network initialization is done using the beacon packet of standard bit length, while the intermediate network update is done using beacon packets of smaller size. The reduction in beacon packet size reduces the transmission time and power consumption. Using selective update procedure, the number of computations required is reduced for finding the optimal energy efficient route to destination. Simulation results show that the proposed scheme can achieve same performance as the existing topology in terms of route length and delivery ratio. Due to reduction in intermediate power consumption and number of computations the overall energy consumption of the network is reduced. Reduction in complexity, computations and energy consumption make the proposed scheme useful for achieving low-power consumption, low cost solutions for IEEE 802.15.4a networks.
\end{abstract}

\section{General Terms}

Energy, cost, computations, packet, algorithm.

\section{Keywords}

Energy efficient topology, Geographical routing, Interference, IEEE 802.15.4a.

\section{INTRODUCTION}

IEEE 802.15.14a / ZigBee standard employs ad-hoc on demand distance vector routing protocol (AODV) and the hierarchical routing protocol (HRP) [1] to achieve low speed wireless communication through simplistic and low cost ubiquitous devices [1]. To reduce to overheads involved in establishing and maintaining the network location based information can be used. Research results show that Geographical routing methodologies achieve better performance than AODV or HRP in presence of location information [2][3][4].

The key aspect in different geographical routing methodologies is the use of greedy algorithm to find the source to destination path with least power, energy or resource consumption. A major bottleneck in the performance of greedy algorithm originated from the obstacles which caused communication interference. Log-distance path model can be used to choose next hop neighbor based on lowest energy/distance value using log-distance path loss model [2][5]. These systems are computationally extensive and may lead to high power consumption. In an alternative scheme the packet reception rate over a considerable period of time is used as a measure to decide next hop neighbor [6][7]. Abrupt or short time change in environmental and transmission conditions can cause un-desirable performance in these algorithms.

Beacon packet based interference aware energy efficient geographical routing algorithm (IEG) can be used for finding next hop neighbor by calculating energy cost along a path [8][9]. It has been shown that as compared to other geographical routing algorithms IEG can adapt to environmental changes with reduced computation expense. However, periodic beacon transmission and computation of next hop energy consumption reduces over-all data transmission rate and increases computations of energy consumption even when the transmission conditions have not changed significantly.

In [10] the modifications in IEG routing are presented to reduce the number of overall computations. It was shown that by selective update procedure the computations required for energy consumption can be avoided. The protocol utilized information sharing method to reduce the computation complexity. The scheme used standard beacon packet for information sharing. Hence, as the number of nodes increased the beacon payload increased thereby increasing beacon transmission energy requirement. Secondly, after establishing the environmental conditions through first set of beacon signals, complete beacon packet transmission is not required for updating node information.

In this paper, Multiple beacon types are introduced in IEG (MbIEG) to reduce the computation complexity. It is shown that after initial information sharing between nodes the network can use beacon packets of smaller packet size to inform the neighboring nodes regarding change in network conditions. Various conditions leading to change in network 
conditions are discussed. Based on these conditions different beacon packet construction is suggested. The simulation result shows that the proposed scheme can achieve same performance as IEG in terms of packet delivery rate and route length with reduced energy consumption. It is shown that as compared to existing protocols the proposed scheme reduces the power consumption by 13 to $20 \%$. In section 2 IEG routing is briefly discussed. In section 3 and 4, the proposed modifications are presented. In section 5, the simulation environment and results are presented, followed by conclusion.

\section{ENERGY-EFFICIENT INTERFERENCE-AWARE GEOGRAPHICAL ROUTING}

\subsection{Interference-Aware Minimum Energy Consumption Estimation}

In [9] and [10], the computations involved in IEG routing are presented. In IEG, information of interference along various paths is used to estimate the minimum transmit power along the route. For a given signal-to-interference-plus-noise ratio (SINR) model, the minimum receiver power threshold, $P_{R X}^{T H}$, is computed by [9]

$P_{R X}^{T H}=10 \log \left(10^{P_{N} / 10}+10^{P_{I} / 10}+10^{\varsigma^{T H / 10}}\right)$

where, $P_{N}$ and $P_{I}$ denotes the noise power and the interference power in $\mathrm{dBm}$; $\zeta^{\mathrm{TH}}$ is the SINR threshold for a successful reception in $\mathrm{dB}$. The noise power depends on hardware used. The minimum power required for successful transmission, $P_{T X}^{\min }$, is given as

$P_{T X}^{\min }(d B m)=P L(d B)+P_{R X}^{T H}(d B m)+\sigma$

where $P L$ is the path loss between the sender and the receiver due to the distance and the obstacles; $\sigma$ is the deviation of the path loss in a fading channel.

In [9], energy efficient version of interference aware routing is presented. The nodes measure the receiver power threshold, $P_{R X}^{T H}$, periodically. The change in interference near a node is sent to other nodes by broadcast mechanism at maximum transmission power, $P_{T X}^{\max }$. The receiver measures the receive power, $P_{R X}$, and determines the path loss as

$$
P L(d B)=P_{T X}^{\max }(d B m)-P_{R X}(d B m)
$$

The total energy, $E$, required for successfully transmitting a data packet is given by

$$
E=E_{T X} \times T_{D A T A}+E_{R X} \times\left(T_{L I F S}+T_{B O}+T_{A C K}+T_{S I F S}\right)
$$

where, $E_{T X}$ and $E_{R X}$ denote the power consumption in $\mathrm{mW}$ in the transmit mode and the receive mode respectively. $T_{D A T A}$, $T_{A C K}, T_{L I F S}, T_{S I F S}$, and $T_{B O}$ denote the durations of the data packet, the acknowledge packet, the long inter-frame space, the short inter-frame space, and the back-off respectively [8]. Proportionality factor $c$, and the power consumption in the transmit mode is proportional to the minimum transmit power $\left(E_{T X} \propto c \bullet P_{T X}^{\min }\right.$ ). Therefore, most of entities in (4) are constant and the energy consumption value is mostly affected by the interference effects when a node transmits a packet.

Using (2) the minimum power for transmission is calculated. For each neighbor, the transmitter computes the total energy, $E$, to transmit the packet from node $a$ to node $b$ using (4). Advance to destination distance, $\mathrm{ADV}$, is calculated as

$$
A D V=d(a, b)-d(c, b)
$$

where, $d(a, b)$ denotes the distance between the node $a$ and the destination $b$. The source node $a$ then transmits the packet to node $c$ with the lowest $E / A D V$. The power and energy requirement increases with interference. Hence, routing with minimum $E / A D V$ minimizes the total energy consumption along the routing path. Fig. 1 shows the basic example involved in IEG.

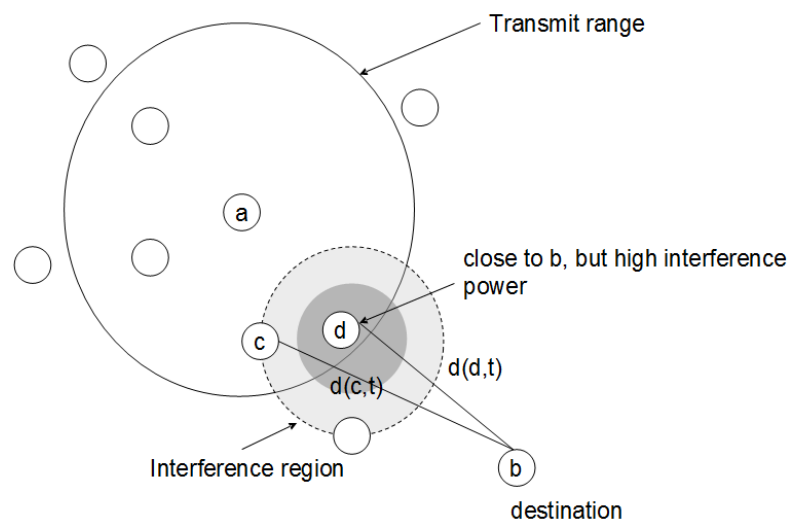

Fig. 1. Interference-aware energy-efficient geographical routing algorithm for IEEE 802.15.4a network (IEG) basic example.

For a network with $m$ number of nodes, in [10] the modified Energy-efficient Interference-Aware Geographical Routing Scheme is presented such that after the initial which can be summarized as

1. Interference and receiver power threshold values are calculated periodically. The energy values are used to establish the routing tables and the beacon packets are transmitted in a pre-defined order.

2. A node $n$, transmits a beacon packet with location information, receiver power threshold value and the minimum required transmission power to all the preceding nodes, 1 to $n-1$

3. Using locally obtained interference information and information from beacon packets all the nodes from $n$ to $m$ calculate the minimum required transmission power from node $n$ to $m$.

4. Nodes finalize their respective routing tables after receiving the beacon packets from all the nodes.

5. For data transmission from node $a$ to $b$, the packet size and estimates the energy consumption, $E$, for each neighbor by using (4). The node $a$ then forwards the packet to the node $c$ with the lowest $E / A D V$ value [9].

6. Packets are forwarded to the neighbor with the positive $E / A D V$ value to prevent the routing loop.

7. When no nodes exists for positive $E / A D V$ value the packet can be transferred to nearest node. 
The procedure is repeated if the interference condition at some node changes and the routing tables of various nodes are to be updated.

A major shortcoming in this scheme is that as the number of nodes, $m$, increases the size of beacon packet payload will increase as additional nodes must add their local information along with the data received from previous nodes. Hence, as more and more nodes transmitted their respective beacon signals the energy consumption in transmission of beacon packets will increase and the time available for transmission of data will be limited.

\section{PROPOSED MULTI-BEACON STRATEGY}

\subsection{Variable Conditions in the network}

In a network of $N$ nodes, in existing IEG routing the broadcasting of beacon packet from first node to other nodes initiates the routing table configuration. All the nodes receive the packet and calculate power and energy values for their respective routing table. Thereafter, next node starts broadcasting the beacon packet. The process continues till all the nodes have transmitted their corresponding beacon packets. This situation is shown in Fig. 2

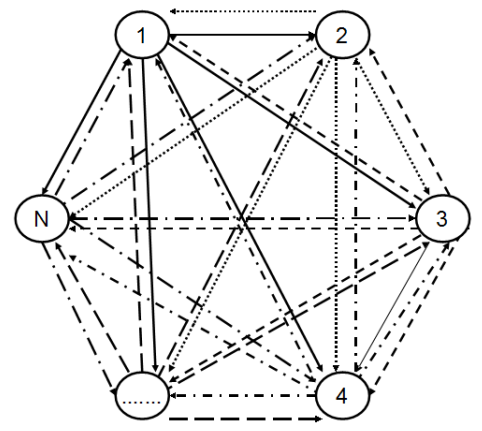

Fig. 2. Beacon packet transfer among nodes in IEG routing algorithm.

To establish the routing path with least energy consumption between two nodes, information of minimum power required for successful transmission, $P_{T X}^{\min }$, and information of interference conditions are required. After initial setup the interference information can change if the location of the node is changed or an interfering object comes in vicinity of the node. The interference caused due to a foreign object effects the $P_{R X}^{T H}$, path loss and is reflected in the deviation of the path loss in a fading channel, $\sigma$. These computations can be done either at transmitter or at receiver. If the deviation of path loss is known to the transmitter, new value of $P_{T X}^{\min }$ can be transmitted as the part of beacon packet. Similarly when the receiver obtains the information from its local resources regarding the deviation in interference power, it can compute new value of $P_{T X}^{\mathrm{min}}$ based on local information and the beacon packet received.

If the node location is changed, the transmitter must send the new node location along with the new value of $P_{T X}^{\min }$. The receiving nodes then update their respective routing tables based on locally calculated path loss value and the information received in the beacon packet.

\subsection{Condition based beacon packets}

After initial network setup, following conditions may require updating the routing tables of various nodes.

1. Local interference conditions are changed but the node location is not changed.

2. Local interference conditions are not changed but the node location is changed.

3. Both local interference conditions and node location are not changed.

Each of these conditions will determine the number of computations that are required for the update of the routing tables at different nodes. When only interference around a node is changes the node can transmit the $P_{T X}^{\min }$ value and the new $P_{R X}^{T H}$ value in the beacon packet. The neighboring nodes can thereby update their table using (4) and the information received from the beacon packet. In this condition the previously determined values of Path loss, $P L$, and path loss variance, $\sigma$, can be used in (4).

In case the node location is changed, new values of of Path loss, $P L$, and path loss variance, $\sigma$, must be calculated by various nodes when the beacon packet is received. Hence, the node whose location is changed transmits the new location along with its $P_{T X}^{\min }$ value and the new $P_{R X}^{T H}$ values. The receiving nodes then use their locally obtained information, combined with data received in beacon packet to calculate total energy, $E$, required for successfully transmitting a data packet to the sending node. This information is then used to update the routing table of various nodes.

When only interference is changed, sub-beacon type 1 is transmitted by node $n$, which is defined as shown below:

$$
\begin{array}{|l|l|}
\hline \Delta P_{R X, n}^{T H}(d B m) & P_{T X, n}^{\min } \\
\hline
\end{array}
$$

Fig. 3. Data segment of Beacon packet broadcast from node $n$.

In the above defined sub-beacon $\Delta P_{R X, n}^{T H}$ refers to difference between the current and previous value of $P_{R X}^{T H}$. Usually the deviation in the $P_{R X}^{T H}$ value is much smaller than its exact value. Hence, the number of bits required for transmission of deviation is much smaller than the bits required for transmission of current value of $P_{R X}^{T H}$. Hence, the difference is transmitted to reduce the beacon payload. The receiving nodes can use the difference in the $P_{R X}^{T H}$ value and the $P_{T X}^{\min }$ value to update their corresponding nodes when only interference is changed.

In case the location of the node is changed, sub-beacon type 2 is transmitted by node $n$, which is defined as shown below:

$$
\begin{array}{|l|l|l|}
\hline \operatorname{Location}(x, y, z) & \Delta P_{R X, n}^{T H}(\mathrm{dBm}) & P_{T X, n}^{\min } \\
\hline
\end{array}
$$

Fig. 4. Data segment of Beacon packet broadcast from node $n$. 
In case the sub-beacon type 2 is transmitted, the receiver uses the location information along with the difference in the $P_{R X}^{T H}$ value and the $P_{T X}^{\min }$ value to calculate the new values of path loss, power and energy required for successful transmission of data packet to the sender. These values are then used to update the routing table of the receivers.

It should be noted that the sub-beacon type 2 can also be used when both interference and location information are changed. Hence, collectively, standard beacon type, sub-beacon type 1 and 2 can identify all the conditions that can possibly exist in the network. As compared to modified IEG of [10], the proposed scheme uses smaller sized beacon packet. Hence, the time, power and energy required for transmission of subbeacon packets is smaller than that of standard beacon packet transmission. Hence, the proposed scheme reduces the power and energy consumption by reducing the packet length during network update cycle.

It should be noted that reduction in number of computation applicable to modified IEG of [10] are applicable to the proposed algorithm also. Hence, by combining the proposed scheme with the modified IEG of [10], the number of computation is also reduced by $50 \%$. Reduction in number of computations reduces power and energy consumption of nodes, while reduced payload in beacon packets reduces transmission power requirements and network bust time due to beacon transmission.

\section{MULTI-BEACON ENERGY- EFFICIENT INTERFERENCE-AWARE GEOGRAPHICAL ROUTING SCHEME}

Based on the previous discussion the proposed Multi-beacon Energy-efficient Interference-Aware Geographical (MbIEG) Routing Scheme can be summarized as

1. Interference and receiver power threshold values are calculated periodically.

2. The energy values are used to establish the routing tables and the beacon packets are transmitted in a predefined order.

3. A node $n$, computes whether the location of the node has been changed.

4. If the location of the node $n$ is changed, the node computes the difference between the new and previous value of $P_{R X}^{T H}$, new value of $P_{T X}^{\min }$ and transmits sub-beacon type 2 .

5. If the location is not changed node $n$ determines if the $P_{R X}^{T H}$ value is changed by more than $2 \mathrm{dBm}$.

6. If the $P_{R X}^{T H}$ value is changed by more than $2 \mathrm{dBm}$ subbeacon type 1 is transmitted.

7. Using locally obtained interference information and information from beacon packets all the nodes from $n$ to $m$ calculate the minimum required transmission power from node $n$ to $m$.

8. Nodes finalize their respective routing tables after receiving the beacon packets from all the nodes.

9. For data transmission from node $a$ to $b$, the packet size and estimates the energy consumption, $E$, for each neighbor by using (4). The node $a$ then forwards the packet to the node $c$ with the lowest $E / A D V$ value [9].

10. Packets are forwarded to the neighbor with the positive $E / A D V$ value to prevent the routing loop.

11. When no nodes exists for positive $E / A D V$ value the packet can be transferred to nearest node.

12. The procedure is repeated if the interference condition at some node changes and the routing tables of various nodes are to be updated.

\section{IMPLEMENTATION AND RESULTS}

NS-2 simulator was used to compare the performance of the proposed MbIEG routing scheme with various existing routing methods. Similar to the path loss model defined in [10] path loss at a distance $d$ is defined as

$$
P L(d)=P L_{0}+10 \eta \log d / d_{0}+X_{\sigma}
$$

where $P L_{0}$ is the path loss at the close-in reference distance $d_{0}$, $\eta$ is the path loss exponent, and $X_{\sigma}$ is a zero mean Gaussian distributed random variable with standard deviation $\sigma$. The hardware dependent values were calculated using the nanoLOC kit which consists of CSS-based NA5TR1 transceiver [11].

Different parameter values used in simulation are shown in Table 1. The power consumption and duration values are drawn from the data sheet of the NA5TR1. Hundred $802.15 .4 \mathrm{a}$ nodes were placed randomly in $300 \times 300 \mathrm{~m}$ plane and two $802.11 \mathrm{~g}$ nodes were placed at $(150,150)$. Two farthest nodes were selected and 100-byte packets were sent from one to another at the rate of 1 packet per 1.5 seconds. On the other hand, MPEG-4 video file with 800-byte packet size and bit rate of $56 \mathrm{kbps}$, was transferred between the $802.11 \mathrm{~g}$ nodes using the Poisson traffic model [12].

Table 1. PARAMETERS USED IN SIMULATION

\begin{tabular}{|c|c|c|c|}
\hline Parameter & Value & Parameter & Value \\
\hline$P L_{0}(\mathrm{~dB})$ & 40 & $E_{R X}(\mathrm{~mW})$ & 82.5 \\
\hline$\sigma(\mathrm{dB})$ & 2 & $T_{\text {LIFS }}(\mathrm{us})$ & 24 \\
\hline$\eta$ & 2.7 & $T_{A C K}(\mathrm{us})$ & 36 \\
\hline$M(\mathrm{~dB})$ & 3 & $T_{\text {SIFS }}(\mathrm{us})$ & 8 \\
\hline$d_{0}(\mathrm{~m})$ & 1 & $P_{N}(\mathrm{dBm})$ & 95 \\
\hline Data rate $(\mathrm{kbps})$ & 250 & $P_{T X}^{\min }(\mathrm{dBm})$ & -33 \\
\hline$E_{T X}(\mathrm{~mW})$ & 75 & $P_{T X}^{\max }(\mathrm{dBm})$ & 0 \\
\hline
\end{tabular}

Similar to the comparison made in [10], the proposed MbIEG can be compared with the greedy algorithm [9]. The proposed MbIEG method does not alter the inherent criteria of choosing the next hop node during transmission. As a result, the greedy algorithm requires smaller routing hops, however, the delivery ratio decreases significantly in greedy algorithm as the interference increases. On the other hand, PRR $\times$ distance algorithm can achieve higher better data delivery ratio as compared to greedy algorithm due to continuous data flow monitoring. However, this leads to higher packet loss since, the PRR $\times$ distance algorithm cannot account for sudden environment changes. The proposed scheme evaluates the change in environment condition using simplified 
computation and reduced complexity similar to mIEG [10]. The proposed MbIEG routing can respond to sudden changes in interference condition. Hence, the proposed scheme can achieve higher data delivery ratio as compared to existing routing techniques. The comparison of proposed MbIEG with existing routing methods with respect to (a) the route length, and (b) the delivery ratio as a function of the interference power is shown in Fig. 5.

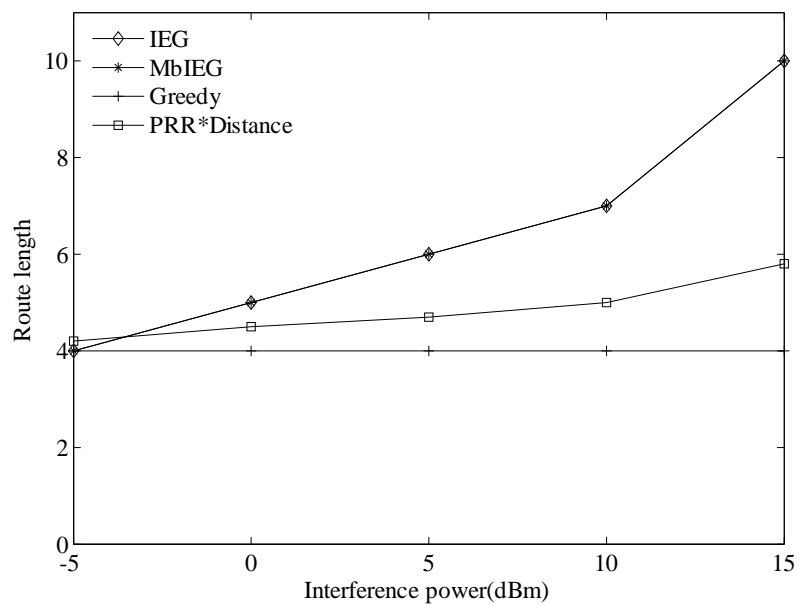

(a)

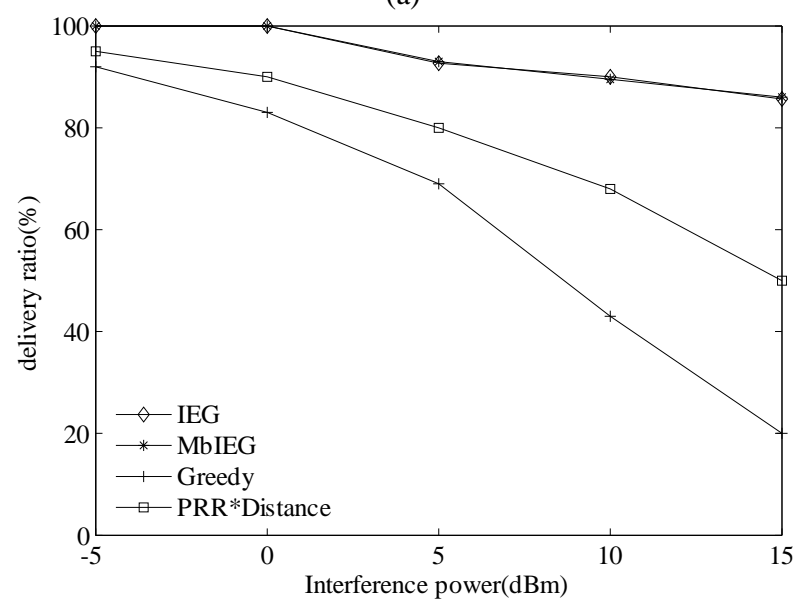

(b)

Fig. 5. Comparison of (a) route length and (b) delivery ratio in proposed MbIEG and various routing algorithms as a function of the interference power

To quantify the effect of reduction in computation the effective over-head for sending a data packet must be considered. These over-heads include the power consumption for computation and maintenance of the routing table in various nodes. In the proposed MbIEG routing, three conditions discussed in section 3 results in different over heads due to difference in beacon packets transmitted in various conditions. Based on these conditions we classify the simulations as

1. MbIEG-C1: Local interference conditions are changed but the node location is not changed.

2. MbIEG-C2: Local interference conditions are not changed but the node location is changed.

3. MbIEG-C3: Both local interference conditions and node location are not changed.
The comparison of data energy consumption per packet is shown in Fig. 6. If only location or interference information is changed, the number of computations required to update the routing table is not high. Hence, in case 1 and 2, the power consumption over heads are lower than that of standard IEG routing. In case both location and interference conditions are changed all the computations required. However, the reduced payload of sub-beacon type 2 results in lower power consumption as compared to the existing IEG algorithm. It is evident that due to reduced payload and computation the power consumption over-head are reduced in proposed scheme in all the conditions.

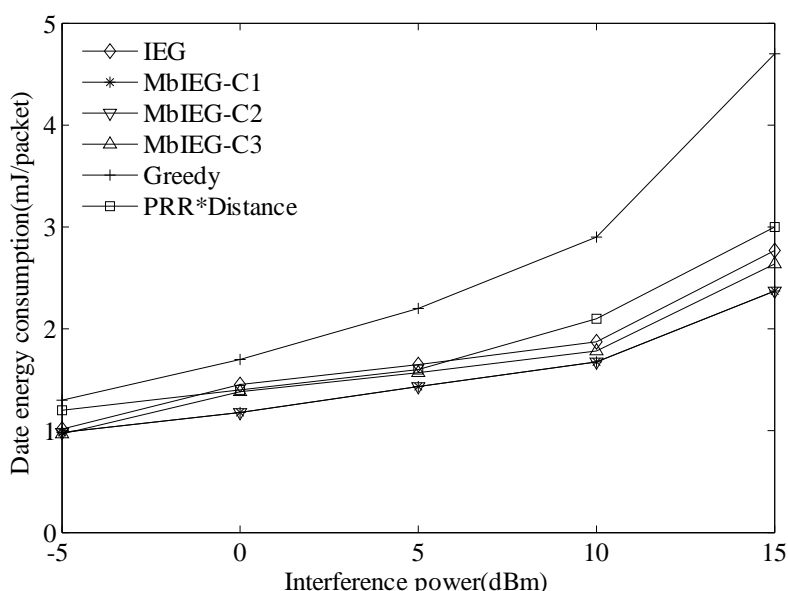

Fig. 6. Comparison of energy consumption over-head in data transmission in proposed MbIEG and original IEG algorithm.

The comparison of energy consumption due to transmission of beacon in IEG and the proposed MbIEG under various conditions is shown in Fig. 7. The simulation results authenticate our hypothesis that the reduction of packet length by using sub-beacon 1 and 2 reduces the energy required for transmission of beacon.

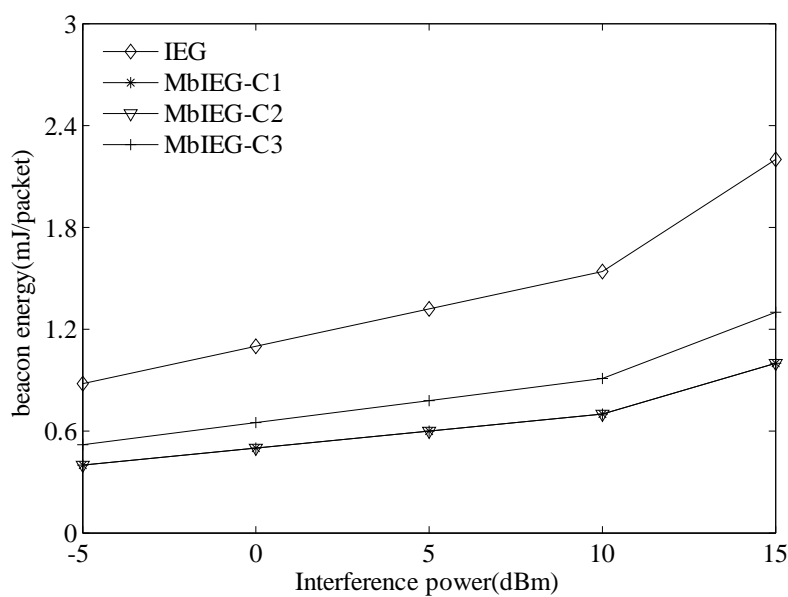

Fig. 7. Comparison of energy consumption over-head in beacon transmission in proposed MbIEG and original IEG algorithm.

Finally, the comparison of total energy consumption in transmission of data and beacons in various interference 
conditions is shown in Fig. 8.

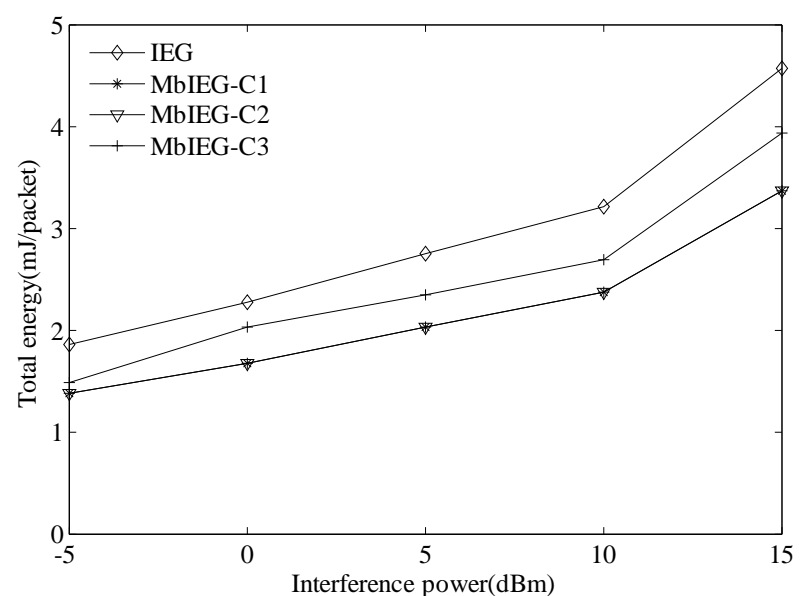

Fig. 8. Total energy consumption in proposed MbIEG and original IEG routing algorithm.

It can be seen that due to reduction in computations, and reduced packet length in beacon transmission, the energy consumption in routing table construction is reduced in proposed scheme. The simulation results presented in Fig. 6, Fig.7 and Fig. 8, shows that the proposed MbIEG routing scheme achieves the same performance as the original IEG with reduced computation complexity and reduced over-all power consumption

\section{CONCLUSION}

In this paper, multi-beacon energy-efficient interferenceaware geographical routing (MbIEG) is presented. It is shown that the original IEG routing requires update in node information when location and/or interference at a given node changes. By identifying these specific conditions, sub-beacon packet structures are proposed. The sub-beacon packets have smaller packet size and support reduced computation strategy of modified IEG routing scheme. Finally, the routing scheme combining the modified IEG and sub-beacon packet transmission is proposed. Simulation results show that the proposed scheme can achieve same performance as original IEG routing with respect to routing length required and data delivery ratio. Furthermore, the proposed MbIEG routing scheme reduces the power and energy consumption over-head per packet. Due to reduced beacon packet size, the power consumption in sub-beacon transmission is smaller as compared that in standard beacon transfer in IEG. The simulation results show that the total energy consumption in proposed MbIEG is lower than that of original IEG routing scheme. Due to reduced power consumption, higher data delivery ratio and reduced data transmission overheads, the proposed MbIEG routing method can be considered as an attractive alternative to various other existing routing methods.

\section{ACKNOWLEDGMENTS}

This research was supported by Basic Science Research Program through the National Research Foundation of Korea(NRF) funded by the Ministry of Education, Science and Technology(2012R1A1A-2012007529)

\section{REFERENCES}

[1] ZigBee Alliance, "ZigBee Specification", online at http://www.zigbee.org, Dec. 2006.

[2] R. Flury, S. Pemmaraju, and R. Wattenhofer, "Greedy Routing with Bounded Stretch", Proc. INFOCOM, Rio de Janeiro, Brazil, Apr. 2009

[3] IEEE computer society, "Part 15.4: wireless medium access control (MAC) and physical layer (PHY) specifications for low-rate wireless personal area networks (WPANS) amendment 1: add alternate PHYs", online at http://standards.ieee.org, Aug. 2007.

[4] B. Karp and H. Kung, "Gpsr: greedy perimeter stateless Routing for Wireless Networks", Proc. MOBICOM, Boston, MA, USA, pp.243-254, Aug. 2000.

[5] R. Veronesi, M. D. Pozzo, V. Tralli, and A. Giovanardi "Energy efficient forwarding strategies for wireless sensor networks in presence of fading and power control", Proc. PIMRC, Berlin, Germany, pp.1383-1388, Sep. 2005.

[6] S. Lee and B. Bhattacharjee and S. Banerjeem "Efficient geographic routing in multihop wireless networks", Proc. MOBIHOC, Urbana-Champaign, IL, USA, pp.230-241, May 2005.

[7] K. Seada and M. Zuniga and A. Helmy and B. Krishnamacharim "Energy-efficient forwarding strategies for geographic routing in lossy wireless sensor networks", Proc. SENSYS, Baltimore, MD, USA, pp.108-121, Nov. 2004.

[8] J. Kim and Y. Kwon, "Interference-aware topology control for low-rate wireless personal area networks", IEEE Trans. Consumer Electron., vol.55, no.1, pp.97104, Feb., 2009.

[9] Junseok Kim and Younggoo Kwon, "Interference-aware Energy-efficient Geographical Routing for IEEE 802.15.4a Networks", IEICE Transaction on Communications, vol.E93-B,no.04, pp.1024-1028, Apr. 2010.

[10] Yuan Hu and Younggoo Kwon, "Reducing Computation Complexity in Interference-aware Energy-efficient Geographical Routing for Low Rate Wireless Personal Area Networks," International Journal of Computer Applications, Vol. 55(12):10-15, October 2012.

[11] STMicroelectronics, "High performance CSS transceiver enabling location awareness", online at http://www.st.com, Sep. 2008.

[12] B. Kim, S. Kim, Y. Fang, and T. Wong, "Two-step multipolling mac protocol for wireless lans", IEEE J. Sel. Area Commun., vol. 23, no. 6, pp. 1276-1286, Jun., 2005. 